\title{
A THERMODYNAMIC APPROACH TOWARDS UNDERSTANDING THE DARK UNIVERSE
}

\author{
Carlos A. Melendres \\ The SHD Institute \\ 216 F Street, Davis, CA 95616, U.S.A. \\ Email: camelendres@SHDInstitute.org
}

December 2018

Keywords:

composition and expansion of the universe, thermodynamics, phase diagram, Quantum Space, spaceons, dark energy, dark matter, cosmological constant, cosmic fluid, Quintessence 


\title{
A THERMODYNAMIC APPROACH TOWARDS UNDERSTANDING THE DARK UNIVERSE
}

\author{
Carlos A. Melendres \\ camelendres@SHDInstitute.org
}

\begin{abstract}
We present a thermodynamic approach in modeling the evolution of the universe based on a theory that space consists of energy quanta and is the cosmic fluid component of the universe. It provides an insight on the nature of dark energy and dark matter, as well as a rationale for the accelerated expansion of the universe.

The universe started from an atomic size volume of an ideal gas at very high temperature and pressure. Upon expansion and cooling, phase transitions occurred resulting in the formation of fundamental particles, and matter. These nucleate and grow into stars, galaxies, and clusters with the aid of gravity. From the cooling curve of the universe we constructed a thermodynamic phase diagram of cosmic composition, from which we obtained a correlation between dark energy and the energy of space. Using Friedmann's equations, our model fits well the WMAP data on cosmic composition with an equation of state parameter, $w=-0.7$. The dominance of dark energy started at $7.25 \times 10^{9}$ years, in good agreement with BOSS measurements. The expansion of space is attributed to Quintessence associated with a quantum space field. Dark Matter is identified as a plasma form of matter similar to that which existed during the photon epoch, prior to recombination.

The thermodynamics of expansion of the universe was adiabatic and decelerating during the first 7 billion years after the Big Bang; it became non-adiabatic and accelerating thereafter. The latter maybe due to an influx of energy from a source outside the universe, if it is open. If it is closed, thermodynamics requires that the pressure of space be negative. Said pressure would cause the accelerated expansion of the universe in accordance with the theory of General Relativity, and the law of conservation of energy. We provide a mechanism to explain this. The acceleration should not be interpreted as due to a repulsive form of gravity. Our Quantum Space model fits well the behavior of the observable universe.
\end{abstract}




\section{INTRODUCTION}

The composition of the universe is a question of great scientific interest. Our universe is essentially dark, consisting of $71 \%$ dark energy, $24 \%$ dark matter and only $5 \%$ ordinary matter, of which $0.5 \%$ is luminous. The nature of dark matter and dark energy remains unknown Dark energy is theorized to cause the expansion of the universe, dark matter is thought to hold the galaxies together . Both remain "mysterious".

The expansion of the universe is intimately related to that of space. The nature of space is not known but much debated [1]. It is generally viewed like a canvas where nature's landscape and events are portrayed; it is then treated geometrically and mathematically as a surface in 4dimensional spacetime. We present a more descriptive model of space based on the theory that it is a quantized dynamical entity which is the "cosmic fluid" that actively participates in the evolution of the universe, along with matter and radiation. The mechanism of evolution proceeds via well known physico-chemical processes. Our model provides an explanation for the accelerated expansion of the universe due to dark energy, and gives an insight on the nature of dark matter.

\section{A MODEL OF SPACE AND COSMIC EVOLUTION}

It has been thought of that space is not truly empty, that "vacuum" contains virtual particles that pop in and out of existence, and is used to explain the Casimir effect [2]. We therefore take the view that space is associated with energy and is quantized. Space consists of energy quanta that propagate as waves described by the Planck quantum energy expression:

$$
\mathrm{E}=\mathrm{hc} / \lambda
$$

The symbols have their usual meaning: $\mathrm{E}$, is the energy; $\lambda$, the wavelength, $\mathrm{c}$, the velocity of light, and $\mathrm{h}$, the Planck constant. The expression defines the equivalence of space (of dimension $\lambda$ ), and energy, in analogy to the relation between energy and mass, given by Einstein's equation, $E=\mathrm{mc}^{2}$ Thus, energy is inherent in space and space in energy. We shall call the quantum units of space as "spaceons". They can be thought of as the carrier of energy and weave the fabric of the universe (consider how a spherical wave of wavelength, $\lambda$, can fill the universe uniformly). From wave- particle duality, the spaceons can also be thought of as a gas which obeys the equation of state [3],

$$
\mathrm{PV}=\mathrm{N}_{0} \mathrm{~K}_{\beta} \mathrm{T}
$$

$\mathrm{P}$ is the pressure of the gas, $\mathrm{V}$ the volume, $\mathrm{T}$ the temperature, $\mathrm{N}$ the Avogadro number and $\mathrm{K}_{\beta}$ the Boltzman constant.

With this theory we can model the gross features of the evolution of the universe as follows: The universe started (at time, $\mathrm{t}=0$ ) as a very small volume of an ideal gas (the spaceons) at an extremely high pressure and temperature. For example, an Avogadro number of gas particles occupying a volume of $4.2 \times 10^{-33} \mathrm{~m}^{3}$ ( a spherical wave of $0.1 \mathrm{~A}^{0}$ radius), at a pressure of $1.976 \times 10^{65} \mathrm{~Pa}$ and a temperature of $10^{32} \mathrm{~K}$. In this initial state of its birth, the universe consisted of "hot" spaceons and radiation in equilibrium. We shall refer to this instant as the Quantum Space Epoch. The spaceons and radiation then expanded and cooled as they propagate and create more space. In the process of cooling to appropriate threshold temperatures, phase transitions occured resulting in the formation of fundamental 
particles, nuclei, and atoms. From the matter formed, gravitation caused the formation of galaxies and stars; these clumped to form clusters, local groups and superclusters. Details of the mechanism involved in the nucleation and growth of matter are not completely known. The universe continues to expand until the present time. The various epochs of the evolution of the universe, in our model, are similar to those of the Big Bang $[4,5,6]$, but without Inflation.

\section{RESULTS AND DISCUSSION}

\subsection{Composition of the Universe}

Several methods are used to determine the composition of the universe. Results of the WMAP satellite studies [7] gave the composition shown in Table I soon after the Big Bang and at present:

Table I-Composition of the Universe

At the Big Bang

Dark Matter

Dark Energy

Ordinary Matter

Neutrinos

Photons
$63 \%$

$1 \overline{2} \%$

$10 \%$

$15 \%$
At Present (13.8 billion years after)

$24 \%$

$71.4 \%$

$4.6 \%$

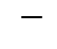

The composition during the cosmic evolution has also been documented $[4,5,6]$.Table II shows this as a function of pressure $(\mathrm{P})$ and temperature $(\mathrm{T})$ at various times and Cosmic Epochs.

Table II. Composition of the Universe During Its Evolution

Time (after Big Bang)/ Temperature (K) Pressure $(\mathrm{Pa}) \quad$ Composition

(Cosmic Epoch)

$<10^{-12} \mathrm{sec} /$

(Quantum Space)

$10^{-12}$ to $0.02 \mathrm{sec} /$

$10^{11}-10^{9} \quad 10^{32}-10^{27}$

I. Fundamental particles

(Quark,Hadron,Lepton,

$>10^{12}$

$>10^{32}$

Hot spaceons + radiation

Electrons,Protons,Neutrons)

0.02 to $300 \mathrm{sec} /$

(Nucleosynthesis)

$300 \mathrm{sec}$ to $3.8 \times 10^{5} \mathrm{yrs} /$

(Photon to Recombination)

$3.8 \times 10^{5}$ to $10^{9} \mathrm{yrs} /$

(Dark Ages to Matter

$\mathrm{S}$ dominated)
$10^{9}-10^{8} \quad 10^{27}-10^{15}$

$10^{8}-10^{3}$

$10^{15}-10^{-11}$

$3 \times 10^{3}-4$

$10^{-11}-10^{-22}$
+ radiation

II. Nuclei of H, He,D,Li

+ radiation

III. Plasma of ionized H, $\mathrm{He}$ and e-

IV.Matter in galaxies,stars space, planets-gases,plasma, solid 
$10^{9}$ to $13.8 \times 10^{9} \mathrm{yrs} /$

(Dark Energy dominated)

$4-2.7 \quad 10^{-22} \ldots \quad$ V. Dark Energy

At temperatures above $10^{12} \mathrm{~K}$ and time $<10^{-12} \mathrm{sec}$, the universe was a primeval hot gas of spaceons and radiation. Upon expansion and cooling, fundamental particles (quarks, leptons, hadrons, protons, neutrons, electrons) were created. On further cooling to about $10^{9} \mathrm{~K}$, nucleosynthesis occurred to produce nuclei of $\mathrm{H}, \mathrm{He} \mathrm{Li}$ and $\mathrm{D}$. At about $10^{8} \mathrm{~K}$ a plasma phase was formed which consisted of electrons and positive ions of $\mathrm{H}$ and He. Radiation (photons, neutrinos) was ever present and dominated the early epoch of the universe. Further cooling of the plasma until about $3 \times 10^{3} \mathrm{~K}$ resulted in recombination of electrons with positive ions of $\mathrm{H}$ and $\mathrm{He}$, converting the plasma to gases. As the universe continued to expand and cool, matter continued to form which, through the action of gravity became stars, galaxies, and clusters. The

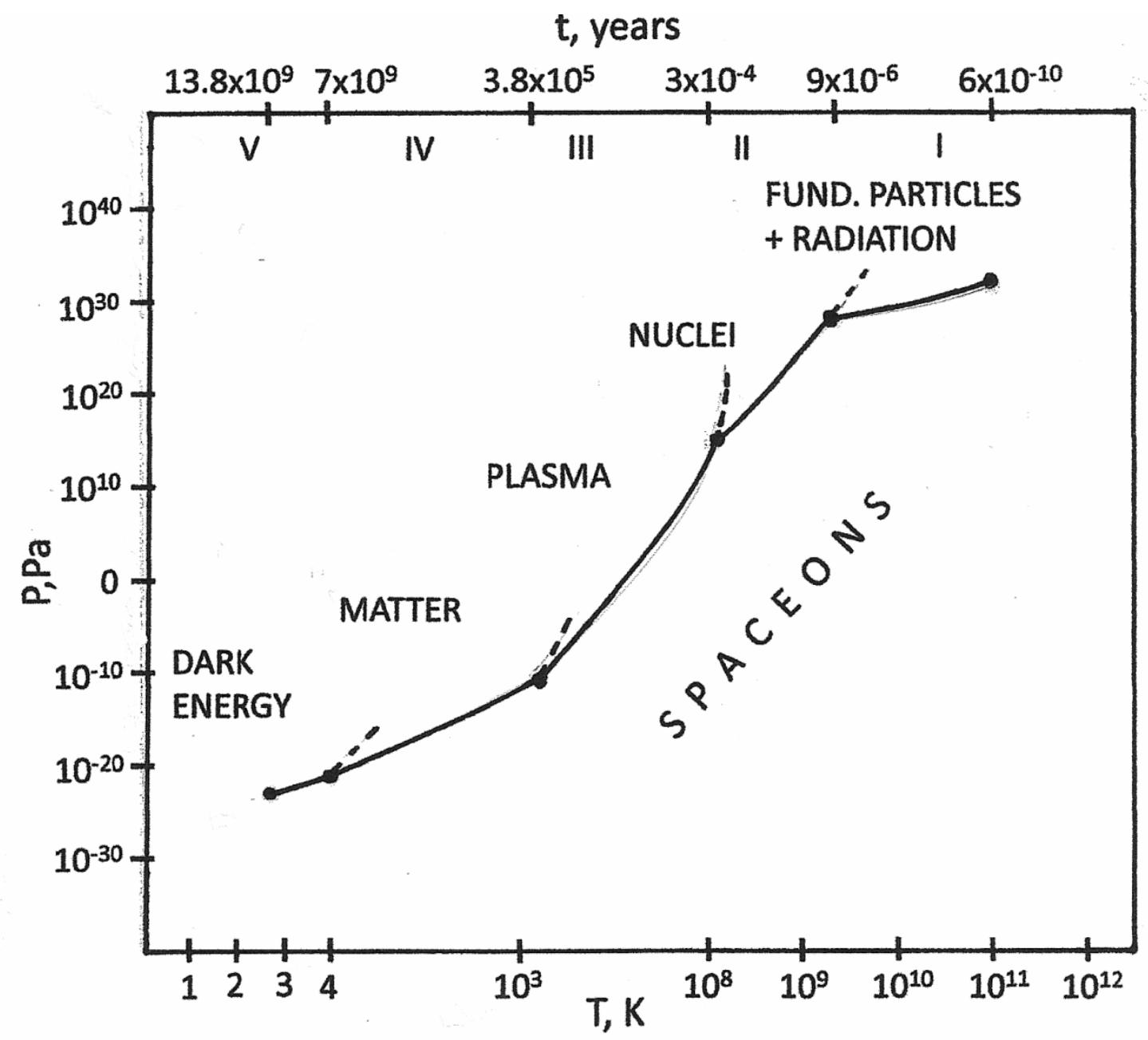

Fig. 1-Schematic Phase Diagram of the Universe with Spaceons

universe cooled to $2.7 \mathrm{~K}$ as indicated by the Cosmic Microwave background (CMB). The primary constituents of the universe are: gases $(\mathrm{H}, \mathrm{He})$, plasma of electrons, protons, and $\mathrm{He}$ ions, ordinary matter (gasses, solids, dust, in stars, galaxies, clusters, intergalactic space), radiation, and spaceons ( gas at all temperatures). 
Thermodynamics is a very powerful method for obtaining compositional information $[3,8]$. From the composition as a function of time, that is, the cooling curve of the universe, we can construct a thermodynamic phase diagram (similar to the well known water phase diagrams with gas, liquid, solid $\mathrm{H}_{2} \mathrm{O}$ of different crystalline forms). While the construction of such diagrams based on cooling curves, is standard practice in the fields of metallurgy and chemistry, for the components of matter, the equivalence of matter and energy, allows one to do the same for the universe as a system. Dark energy and dark matter are components of the universe. We have thus constructed a thermodynamic phase diagram $[3,8]$ for our model universe with spaceons, from the cooling curve of the universe represented by the data in Table II.

The result is shown in Fig.1. (Note that the figure is schematic and the temperature is not drawn to scale in order to highlight the phases formed). The major phases are: matter (IV, solid, gasses, plasma formed in galaxies, stars, clusters), a plasma phase (III) formed immediately after nucleosynthesis, dark energy (V) and spaceons (gas). The broken lines indicate the overlap between various phases during the process of formation. Dark matter is not yet indicated since its nature remains unknown. All phases are in contact with spaceons at all epochs of cosmic evolution. This is as it should be since space is in contact with all elements of the universe at all times. At extremely high temperatures and pressures (right end), fundamental particles and radiation are indistinguishable from the hot spaceons; while at low temperature/pressure (left end), dark energy overlaps with the cold spaceons. These end points are critical points, where 2 phases co-exist. In the case of water, for example, at the critical temperature of $647 \mathrm{~K}$ and pressure of $2.2064 \times 10^{7} \mathrm{~Pa}$, liquid water is indistinguishable from its vapor phase.

\subsection{Dark Energy and the Expansion of the Universe}

Dark energy constitutes $71 \%$ of our universe. It is hypothesized to be an unknown form of energy that permeates all of space uniformly. It is invisible and difficult to study because it does not interact with radiation, hence cannot be investigated spectroscopically. It only interacts with gravity. Its density is very low, less than ordinary and dark matter, it converts to dark matter, was less in the past than at present, and it is thought to function like an anti-gravity force. It is believed to cause the accelerated expansion of the universe $[9,10,11]$.

Based on our model, the expansion of the universe could be thought of as the expansion of spaceons into the void ( "nothingness"). This appears to be a perfectly natural process driven by a pressure gradient between the "filled" space and the void. It can also be thought of as driven by the pressure and temperature differential of the hot (high energy, short $\lambda_{\mathrm{s}}$ ) and cold (low energy, long $\lambda_{s}$ ) gas states. It is an inherent property of space that it needs to expand in order to attain a lower energy state (longer $\lambda_{s}$ ). The expansion may be viewed from the standpoint of Quantum Field Theory [12], as arising from a force that is associated with a field. The latter is the space field from which emanate space quanta, the spaceons. (This is analogous to the gravitational field with its associated gravitational force.) It is a scalar field, dubbed "Quintessence", that has been theorized to be the substance which comprises Dark Energy [10].

One can see from our thermodynamic phase diagram (Fig.1) that dark energy is a phase that overlaps with a new entity that we have introduced as a component of the universe, i.e., the spaceons; the two phases are indistinguishable. Hence, dark energy can be associated with spaceons, the energy of space. The amount of dark energy soon after the Big Bang was relatively small (Table I) because most of the energy of space was converted to radiation, 
fundamental particles, dark matter and ordinary matter. Its density remains low as the universe expands.

The behavior of our model universe with spaceons is well suited for mathematical treatment using the Friedmann-Lemaitre-Robertson-Walker (FLRW) metric [11] .The second Friedmann equation [10,11] delineates the contributions of the various components of the universe (matter, radiation, and vacuum) to the acceleration of expansion of the universe, i.e.,

$$
\mathrm{d}^{2} \mathrm{a} / \mathrm{dt}^{2}=-(8 \pi \mathrm{Ga} / 3)\left\{(1 / 2) \rho_{\mathrm{m}}+\rho_{\mathrm{r}}-\Lambda / 8 \pi \mathrm{G}\right\}
$$

We have neglected the curvature term , $\mathrm{k}$, normally appearing in 3), by adopting a model of simple Newtonian gravity rather than Einstein's spacetime model. In the above equation, a is the scale parameter, $G$ the gravitational constant; $\rho_{\mathrm{m}}$ is the mass-energy density of matter, $\rho_{\mathrm{r}}$ that of radiation, and $\Lambda$, the cosmological constant which represents the energy of the vacuum [10]. Based on our model of space, "vacuum energy" is no longer a meaningful term to use; it is preferable to use the term "energy of space". The cosmological constant will then be replaced by the energy density of space, i.e.

$$
\mathrm{d}^{2} \mathrm{a} / \mathrm{dt}^{2}=-(8 / 3) \pi \mathrm{Ga}\left\{(1 / 2) \rho_{\mathrm{m}}+\rho_{\mathrm{r}}+\left(\rho_{\mathrm{s}}+3 \mathrm{P}_{\mathrm{s}}\right)\right\}
$$

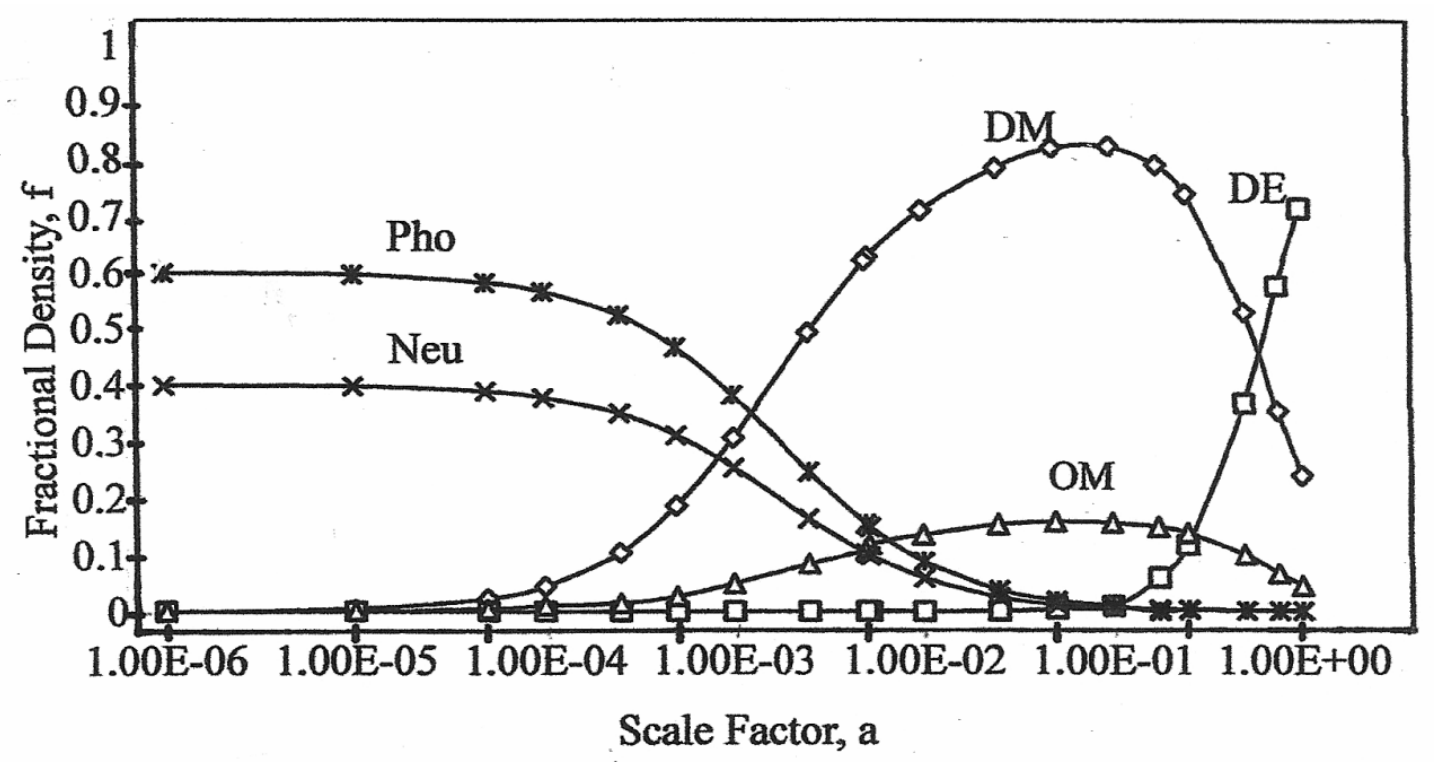

Fig.2- Fractional Energy Density (f) vs Scale Factor (a), fit with $w_{s}=-0.74$

DE-Dark Energy; DM-Dark Matter; OM-Ordinary Matter; Pho-Photons; Neu-Neutrinos

and $\rho_{\mathrm{s}}$ is the energy density of space. It is related to the pressure $P_{\mathrm{s}}$ via the equation of state $\mathrm{P}_{\mathrm{s}}=\mathrm{w}_{\mathrm{s}} \rho_{\mathrm{s}}$ where $\mathrm{w}_{\mathrm{s}}$ is the equation of state parameter. A negative pressure would give rise to a positive term for the contribution of $\rho_{\mathrm{s}}$ that can cause an acceleration of the expansion.

For the purpose of fitting the observed data (Table I), it is preferable to use another form of the Friedmnan equation. It is common to utilize one involving the Hubble constant, H, which is measured in experiments and to use velocity, da/dt, rather than acceleration $[9,10,11]$. Thus, 


$$
a H^{2}=(d a / d t)^{2} a^{2}=\left(H_{0}\right)^{2}\left[\Omega_{m} a^{-3}+\Omega_{r} a^{-4}+\Omega_{s} a^{-3(1+w s)}\right]
$$

$\mathrm{H}=(\mathrm{da} / \mathrm{dt}) . \mathrm{a}, \mathrm{H}_{0}$ its value at the present time; $\varrho_{i}$ is the density parameter for component $\mathrm{i}, \varrho_{c}$ is the critical energy density of the universe (the density of the universe at the present time) and $\Omega_{i}$ $=\varrho_{i} / \varrho_{c}=f$, the fractional energy density.

A plot of fractional energy density as a function of of "a" can be constructed to fit the measured composition of the universe at about the time of the Big Bang and at the present time (see Table 1)

The plot in Fig. 2 shows the evolution of the composition of the universe. The dominance of radiation, matter, and dark energy at different times are amply illustrated. As can be seen there is good fit of the data summarized in Table I at time $\mathrm{t}=13.8$ billion years $(\mathrm{a}=1)$ and at the time of the Big Bang, at about $\mathrm{t}=380,000$ years $\left(\mathrm{a}=5.25 \times 10^{-3}\right.$, redshift $\left.\mathrm{z}=1089\right)$, with an equation of state parameter, $w_{s}=-0.7$. Dark Energy is very small in the early universe as most of the energy is used in the creation of radiation and particles. As the universe expands, however, the energy densities of radiation and matter continue to dilute and are eventually overcome by the energy density of Dark Energy. This occurs at around 7 billion years at which time the expansion of the universe starts to accelerate. This result has been established by baryon acoustic oscillation measurements in the BOSS project $[13,14]$ and supernova measurements [18] . Fig.3 lends support to these findings. One sees that the energy density of Dark Energy (DE1) crosses that of the total energy (TM1) of matter at $\mathrm{a}=0.65\left(\mathrm{t}=7.25 \times 10^{9}\right.$ years $)$.

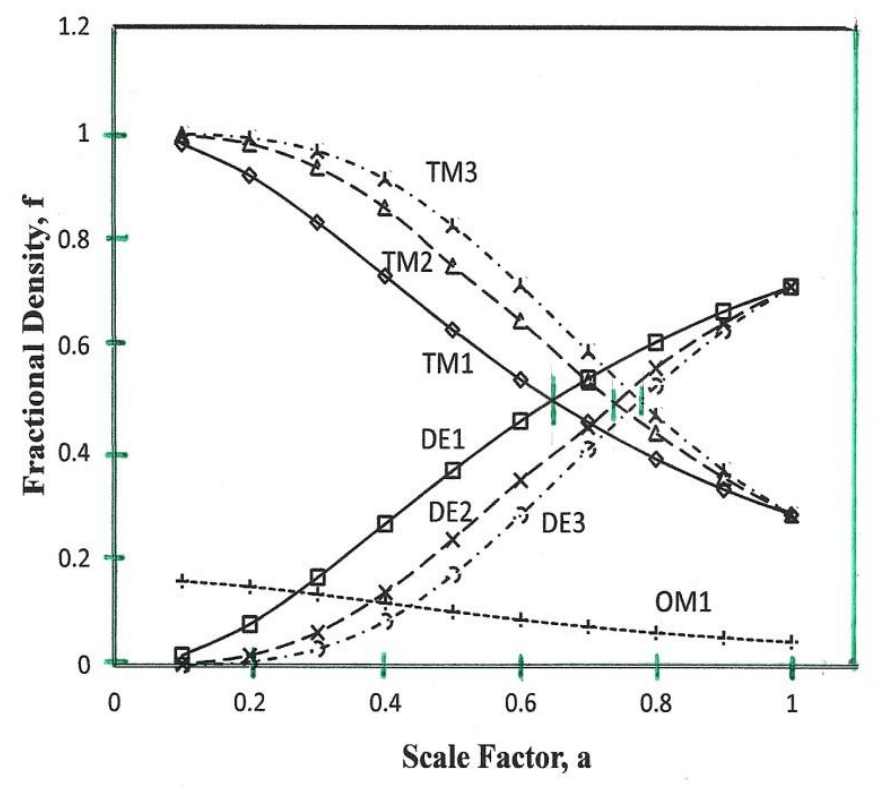

Fig.3- Fractional Energy Density (f) vs Scale Factor (a)

DE1- Dark Energy, $w_{s}=-0.7$; DM1-Dark Matter;

OM1-Ordinary Matter;TM1 - Total Matter; DE2, TM2 for

it

fit with cosmological constant, $w=-1$; TM3, DE3-for fit

with Phantom Energy model, $w=-1.2$ 
Siegel [15] has obtained a similar fit using a cosmological constant but did not give a value for the transition time. Our calculation with a cosmological constant $(\mathrm{w}=-1)$ yielded a time of $8.75 \times 10^{9}$ years $(\mathrm{a}=0.74)$ for the dominance of dark energy (Fig. 3, broken line, DE2, TM2). Result for $w=-1.2$, corresponding to the so-called Phantom Energy model [16 ] yields an even later transition time of $9.2 \times 10^{9}$ years (DE3, TM3). The measurement of transition time appears to be a good test for the suitability of a model for Dark Energy.

The concept of a constant energy density for space leads to some difficulties. The cosmological constant is considered to be equal to the energy of the vacuum. Calculations give a 120 orders of magnitude for the calculated value of the density of dark energy; this is clearly catastrophically wrong. Moreover, the constancy of dark energy in the course of cosmic evolution is also thought to be unlikely [17]. It also poses difficulties in answering the question of where the energy comes from during the expansion of the universe. It is more reasonable to expect that the energy density would dilute as the universe expands for all forms of energy (Fig 4). Quintessence, with spaceons as the cosmic fluid that is a component of the universe, appears to be the better explanation for dark energy.

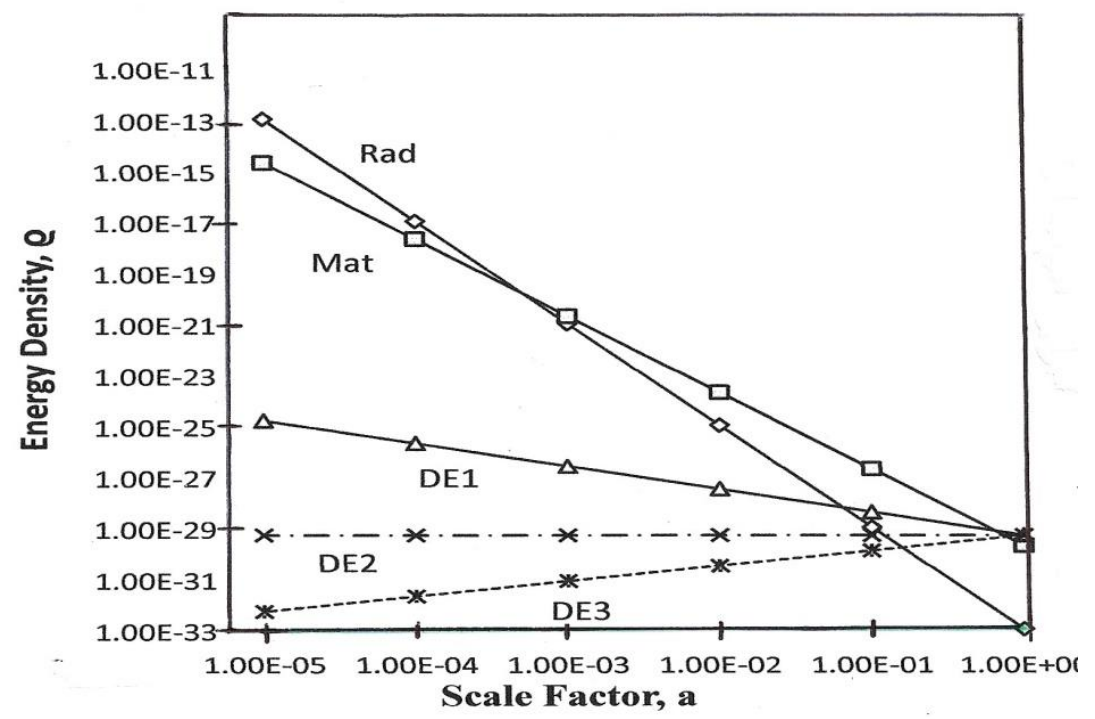

Fig 4. Energy Densities $\left(\rho, \mathrm{g} / \mathrm{cm}^{3}\right)$ vs Scale Factor (a) for 3 models of Dark Energy;Rad-total radiation; Mat-total matter; DE1-Dark Energy with $\mathrm{w}_{\mathrm{s}}=-0.7$; DE2-w $=-1.0($ Cosmological Constant); DE3-w =-1.2 (Phantom Energy Model)

\subsection{THERMODYNAMICS OF EXPANSION AND ACCELERATION}

In our model, the universe started with a finite amount of energy. Expansion was assumed to be adiabatic), i.e. no energy transfer in and out of the universe (also constant Entropy, dS=0. From thermodynamics,

$$
\mathrm{Q}=\mathrm{dE}+\mathrm{PdV}=0,
$$

where $\mathrm{Q}$ is the energy flowing into or out of the system, $\mathrm{dE}$ the change in internal energy, $\mathrm{P}$ the pressure, and $\mathrm{dV}$ the change in volume during the expansion. The work to create space, $\mathrm{PdV}$, is done at the expense of the internal energy,i.e $\mathrm{dE}=-\mathrm{PdV}$ or 


$$
\mathrm{dE} / \mathrm{dV}=-\mathrm{P}
$$

the slope of the $\mathrm{dE} / \mathrm{dV}$ plot is negative. One can also derive the relation,

$$
\begin{gathered}
H .=\left(1 / 4 \pi r^{3}\right)[(d V / d E)](d E / d t) \text { or } \\
d E / d t=4 \pi r^{3} H[d E / d V]
\end{gathered}
$$

where, $\mathrm{r}$, is the radius of the universe, and $\mathrm{H}$ is the Hubble constant, proportional to expansion velocity. To a first approximation, a decrease in $\mathrm{H}$ indicates a decrease in internal energy of the universe with time,t. BOSS studies of Busca et al [13, Fig 21.], show the expansion of the universe to be slowing down during the first 6 billion years after the Big Bang This is consistent with our model universe with the expansion being adiabatic initially, i.e. $\mathrm{dE} / \mathrm{dt}=-$. While it is not very clear, from the scatter of the data in [13], unpublished figure by a Berkeley group [14] shows that it reached a minimum around 7.5 billion years. Turner and Riess [18] have also shown that the universe changed from deceleration (during the matter dominated epoch) to acceleration at a redshift, $\mathrm{z}=1$ or $\mathrm{t}=7 \times 10^{9}$ years. The expansion velocity increased thereafter. The slope $\mathrm{dE} / \mathrm{dV}$ (and that of $\mathrm{dE} / \mathrm{dt}$ ) became positive at large $\mathrm{z}$.

Thermodynamically,

$$
\mathrm{dE} / \mathrm{dV}=(\mathrm{dQ} / \mathrm{dV})-\mathrm{P}=+
$$

that is, $\mathrm{Q}$ is no longer zero; the expansion has become non-adiabatic; this means an ingress of energy from outside the universe. Thermodynamics thus points to the possibility that the accelerated expansion of the universe occurred with an "injection" or "leak"of energy from outside the universe, if it is open. However, there is no evidence at the present time that "something" exists outside the universe to support this.

Our model is for a closed universe. Thus the expansion must remain adiabatic. Thermodynamics then demands that for the accelerated expansion to continue; the Pressure, $\mathrm{P}$, in equation (10) must be negative. It is interesting to note that this is just what is required by the Theory of Relativity (in the second Friedmann equation , 4 above). It is also the negative pressure associated with Dark Energy. This coincidence is truly amazing! However, it is important to note that the negative pressure cannot be taken as due to a repulsive form of gravity, as is often theorized.

We try to answer the question of where the energy comes from to sustain the accelerated expansion by dark energy. The negative pressure means work is done on the system and energy is added to it. A likely source is the gravitational field through the action of gravity. One can consider that gravity exerts an attractive force to shrink space and causes its energy to increase. In analogy, one can imagine a rubber hose filled with running water. Squeezing the hose momentarily shrinks its diameter and causes water to squirt out. Fig. 5A shows schematically the state of the universe during the period of deceleration $\left(5-7 \times 10^{9} \mathrm{yrs}\right)$. Consider the cosmic fluid flowing through a tube (dot-dash lines) within the universe. The Hubble flow (HF) is slowing down until it comes nearly to a halt. The pressure of the universe is outward $\left(\mathrm{P}_{\mathrm{s}}=+\right)$ due to the 
inherent property of space (the spaceons) to expand into the void; the slope $\mathrm{dE} / \mathrm{dV}$ is negative (equation 7). In Fig 5B, at the transition point, the attractive force of gravity $\left(\mathrm{F}_{\mathrm{g}}\right)$ starts to squeeze the universe and exerts a pressure inward $\left(\mathrm{P}_{\mathrm{g}}=-\right)$; now $\mathrm{dE} / \mathrm{dV}$ is positive. In the process, the "cosmic fluid" contained in the tube is squeezed out much like that of the rubber hose. The fluid velocity or the Hubble flow increases, manifesting itself as an accelerated expansion of the universe. Note, however, that while the "expansion" of the universe appears to accelerate, it might be that the action of gravity has started to shrink it. This could be the beginning of the contraction of the universe to return to its state before the Big Bang, i. e. the so called Big Crunch [19] of a cyclic universe .Finally, it is interesting to note that our Quantum Space model nicely depicts the behavior of our observable universe.

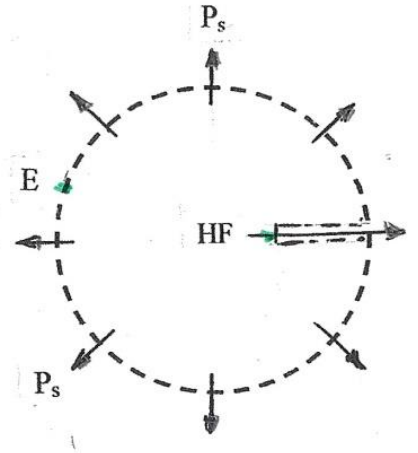

A

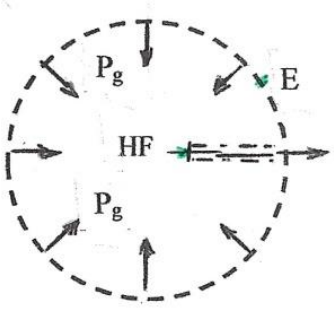

B

Fig 5. Schematic of the Universe during the period

of deceleration (A) and acceleration (B)

$\mathrm{P}_{\mathrm{s}}=+$, pressure due to space; $\mathrm{P}_{\mathrm{g}}=-$, pressure

due to gravitational force; $\mathrm{HF}=$ Hubble flow;

$\mathrm{E}=$ edge of the universe

\section{ON THE NATURE OF DARK MATTER}

Finally, we say some words about the other component of our invisible universe, dark matter; its known characteristics match well those of a component present in our Phase Diagram (Fig.1). Dark matter [20] comprises $84 \%$ of the total mass of matter in the universe. At present, it makes up $24 \%$ of the total mass-energy of the universe It remains a mysterious, hypothetical form of matter.

Among properties that have been reported in the literature are:

1). it neither emits or absorbs electromagnetic radiation, hence it is difficult to study.

2). it moves without friction.

$3)$. it can only be detected through its gravitational effects on the motion of galaxies.

4). it is spread over large areas, like a cloud, and forms a "halo" around galaxies and clusters; its density decreasing as one moves away from the center [21].

5). it is also found in filaments between galaxies and clusters [22]. It has been observed that ordinary matter traces the path of dark matter; this has been attributed to a strong interaction between ordinary matter and dark matter.

We proceed with the premise that all components of the universe were formed during the cosmic evolution and would have left their footprints in the sands of time, e.g, the CMB. The phase diagram (Fig.1) shows that the major phases in the formation of the universe are dark 
energy, ordinary matter (gasses, solids), and plasma. Any present-day component of the universe must originate from one of these phases. From the observed properties of dark matter, and known characteristics of plasma [23], it appears reasonable to make the assignment that dark matter corresponds to the plasma form of matter that existed during the photon epoch, just before recombination. At this time the universe was opaque due to scattering of all photons by free electrons and protons. The following facts support this contention:

1). WMAP data, mentioned earlier, show that dark matter constitutes the major component of the universe soon after the Big Bang. During this time, the universe consisted of a hot plasma of electrons, and ionized $\mathrm{H}$ and $\mathrm{He}$. It was opaque and cannot emit or absorb light. This state persisted until about 380,000 years after the Big Bang at which time recombination took place and the universe became transparent.

2). Other characteristics of plasmas match exactly those of dark matter. Plasma, like dark matter, hangs around like a cosmic fog around galaxies and clusters, making it invisible and difficult to characterize.

3). That ordinary matter traces the path of dark matter can be explained by the fact that upon recombination of electrons and positive ions in the dark matter plasma, ordinary matter is formed; the plasma evaporates into $\mathrm{H}$ and He gasses. Thus ordinary matter follows the trail of dark matter.

4). Filamentation is a characteristic of plasmas; they move without friction, since the ions do not have attractive interaction and move collectively instead [23]. This lack of interaction also explains the origin of dark matter halos [21,22] that hover around galaxies.

5). The dark matter plasma scatters elastically and hence do not clump or "stick together" thus remaining diffuse, fluffy, and"halo-like". Hence, galaxies cannot form directly from dark matter.

Energy exchange between the dark matter plasma and the surrounding hydrogen has been observed to provide a mechanism for energy exchange between the two states of matter [24]. This is consistent with (3) above. Finally, plasma constitutes the major form of matter in the universe [23], most of it invisible. Dark matter being a plasma form of matter seems closer to reality than exotic particles, like WIMPS, MACHOS, etc. [25] whose existence have not been demonstrated.

\section{.5). CONCLUSION}

A thermodynamic approach, using a Quantum Space model, appears useful in understanding the composition and evolution of our dark universe. Dark energy is the energy of space and the cosmic fluid that is the component of the universe responsible for its expansion. It is associated with a scalar field, dubbed as Quintessence. Dark matter, on the other hand, is a plasma form of matter, similar to the state of the universe at the photon epoch, before recombination. It is the precursor in the formation of ordinary matter. Dark energy and dark matter are neither "dark" nor "mysterious", they are just invisible; one is transparent, while the other is opaque. Further work is necessary to better understand the nature and properties of the quantum space field and spaceons. We also need to understand better plasma in space and dark matter halos. Our model brings into question some long standing concepts in the physical sciences. The concept of vacuum appears outdated; the term "vacuum energy" should be more appropriately called "space energy". The Theory of Inflation, also needs to be reexamined; it 
appears unnecessary to produce a homogeneous and isotropic universe.. Finally, thermodynamics indicates that the acceleration in expansion of our universe maybe related to a non adiabatic process due to an influx of energy from "outside" the universe, if it is open. If it is closed, the acceleration would continue, with a negative pressure as thermodynamically required for energy conservation, consistent with the Theory of General Relativity and Friedmann's equation. We provide a mechanism to explain the acceleration in Hubble Flow. It should not be interpreted as due to a repulsive form of gravity. Our Quantum Space model fits well the behavior of the observable universe.

\section{REFERENCES}

1. Futch, M., Leibniz, Metaphysics of Time and Space, Springer, NY (2008)

2. Jaffe, R.L, The Casimir Effect and the Quantum Vacuum. in: Physical Review D. 72, (2005)

3. Atkins, P. and De Paula, J. Physical Chemistry, W.H.Freeman, NY (2014)

4. Silk, J. The Big Bang, W.H. Freeman, N.Y. (1989)

5. Weinberg,S., The First Three Minutes, Basic Books, NY (1977)

6. Delsemme, A., Our Cosmic Origins, Cambridge University Press,UK (1998)

7. Bennett, C.L.; Larson, L.; Weiland, J.L.; Jarosk, N.; et al. "Nine-Year Wilkinson Microwave Anisotropy Probe (WMAP) Observations: Final Maps and Results". The Astrophysical Journal Supplement. 208 (2): 20 (2013)

8. Kirkwood, J.G. and Oppenheim, I. Chemical Thermodynamics, McGraw-Hill, NY. (1961)

9. Frieman, J. A.; Turner, M. S.; Huterer, D. "Dark Energy and the Accelerating Expansion of the Universe". Annual Review of Astronomy and Astrophysics. 46 (1): 385-432 (2008)

10. Stenger, V.J., The Comprehensible Cosmos, Prometheus Books, N.Y. (2006)

11. Weinberg, S., Cosmology, Oxford University Press, Oxford, UK (2014)

12. Zee, A., Quantum Field Theory in a Nutshell, $2^{\text {nd }}$ edition, Princeton Univ Press, NJ (2010).

13. Busca, N.G.; Delubac, T; Rich, J., et al, "Baryon Acoustic Oscillations in the Ly- $\alpha$ Forest of BOSS Quasars", Astronomy and Astrophysics, 552, A96 (2013)

14. in https://scitechdaily.com/scientists-use-quasars-to-probe-dark-energy-measure-its-role-inthe-evolution-of-the-universe/

15. Siegel, E., personal communication, August 2016

16. Caldwell, $R$. R. "A phantom menace? Cosmological consequences of a dark energy

component with super-negative equation of state". Physics Letters B. 545 (1-2): 23-29. (2002).

17. Zlatev, I.; Wang, L.; Steinhardt, P. "Quintessence, Cosmic Coincidence, and the

Cosmological Constant". Physical Review Letters. 82 (5): 896-899 (1999)

18. Turner, $M$ and Riess, A "Do Type Ia Supernovae Provide Direct Evidence for Past

Deceleration in the Universe?", Astrophysical Journal, 569, 18-22, (2002)., also in Ries, A and Turner, M., Scientific American, February 2004, p 62

19. Davies, P The Last Three Minutes: Conjectures About The Ultimate Fate Of The Universe, Basic Books, (1997).

20. Trimble, V. "Existence and nature of Dark matter in the universe". Annual Review of Astronomy and Astrophysics. Palo Alto, CA, Annual Reviews, Inc. 45: 425-472 (1987).

21. De Rujula, A, Jetzer, P, Masso, E, "On the Nature of the Dark Halo of Our

Galaxy”,Astronomy and Astrophysics, 254, 99, (1992) 
22. Dietrich, J., et al, “A filament of dark matter between two clusters of galaxies ", Nature 487, 202-204 (2012).

23. Gurnett, D.A.; Introduction to Plasma Physics: With Space and Laboratory Applications, Bhattacharjee, A., Cambridge University Press, UK (2005)

24. Bowman, J.D., Rogers, A.E, Monsalve, R.A. et al, "An absorption profile centered at 78 megahertz in the sky-averaged spectrum", Nature 555, 67 (2018)

25. Griest, Kim. "The Search for the Dark Matter: WIMPs and MACHOs". Annals of the New York Academy of Sciences. 688, 390-407 (1997) 